\title{
Somatotopic Mapping of the Primary Motor Cortex in Humans: Activation Studies With Cerebral Blood Flow and Positron Emission Tomography
}

\author{
SCOTT T. GRAFTON, ROGER P. WOODS, JOHN C. MAZZIOTTA, AND MICHAEL E. PHELPS \\ The Division of Nuclear Medicine and Biophysics, Department of Radiology; The Laboratory of Nuclear Medicine; and \\ The Department of Neurology, University of California at Los Angeles School of Medicine, Los Angeles, California 90024
}

\section{SUMMARY AND CONCLUSIONS}

1. The somatotopic representation of the human primary motor cortex was examined noninvasively with estimates of cerebral blood flow (CBF) obtained with positron emission tomography. Twelve normal subjects performed a motor tracking task with the arm, first finger, tongue, and great toe commensurate with the bolus injection of radioactive $\mathrm{H}_{2}{ }^{15} \mathrm{O}$. Images of the relative percent increase of blood flow, compared with control studies, demonstrated reproducible foci of CBF increases in the motor cortex in every subject. Each motor task could be localized to a predictable site on a coronal section containing the precentral gyrus.

2. In reproducibility experiments of repeated measures, it was determined that two foci of activation in the primary motor cortex could be discriminated with a $95 \%$ confidence if they were separated by $5.4 \mathrm{~mm}$.

3. In five subjects with matched magnetic resonance imaging studies, the sites of activation were variable with respect to surface anatomy and could be found at the depth of sulci or the surface of gyri. The findings were similar to previously reported electrophysiological studies using direct cortical stimulation.

4. The method may be applied to the in vivo functional mapping of the primary motor cortex in patients with cerebral disorders.

\section{INTRODUCTION}

Current understanding of the somatotopic representation of the primary motor cortex may be traced to the pioneering experiments of David Ferrier. Drawing on stimulation techniques developed by Hitzig, Ferrier used direct application of low-current Faradic stimulation to the cerebral cortex in the region of the central fissure in the macaque monkey (Fritsch and Hitzig 1870). He was able to demonstrate a predictable correlation between stimulation site and motor response (Ferrier 1875, 1876). Stimulation close to the interhemispheric fissure induced both simple and complex movements of the leg. More ventral stimulation induced movements of the upper extremity. Stimulation close to the lateral fissure induced movements of both the tongue and mouth. These observations were expanded by Sherrington, among others, who examined the distribution of the motor responses along the central fissure in several anthropoid species and summarized his findings in 1917 (Leyton and Sherrington 1917). He was able to demonstrate the same sequential pattern of homuncular responses and to localize motor responses to the precentral gyrus. He also attempted to associate specific motor responses from stimulation of the precentral gyrus to regional gyral anatomy near the central fissure, concluding that "their use for strict localization is small" (Sherrington 1906).

Detailed confirmation of the somatotopic arrangement of the motor cortex in human stems from the systematic work of Penfield, who performed direct cortical stimulations in awake patients (Penfield 1958; Penfield and Boldrey 1938; Penfield and Jasper 1954). Although Penfield could demonstrate a highly reproducible sequence of responses along the motor cortex, he noted a wide variation between individuals in the exact site and amount of motor cortex associated with limb movements. In his patient population as a whole, for example, hand movements could be elicited by the stimulation of motor cortex spanning 5.5 $\mathrm{cm}$. His patients suffered from a variety of neurologic disorders, including space-occupying lesions, however. The degree to which these pathological conditions might have altered the normal distribution of responses in the motor cortex is not known.

The goal of the current study was to develop a noninvasive in vivo technique with positron emission tomography (PET) for the systematic examination of the somatotopic organization of the primary motor cortex in normal humans. Previous imaging studies using inhaled Xenon-133 with external detectors or emission tomography.as well as PET imaging have demonstrated focal increases of cerebral blood flow (CBF) as well as oxygen and glucose metabolism during the performance of a variety of motor tasks (Ingvar and Philipson 1977; Lauritzen 1981; Mazziotta 1985; Mazziotta and Phelps 1985; Mazziotta et al. 1985; Orgogozo and Larsen 1979; Orgogozo et al. 1979; Roland 1984; Roland et al. 1980a,b; Roland et al. 1981). A detailed examination of the somatotopic distribution of these responses in the motor cortex has never been reported. Somatotopy has been examined in the primary sensory cortex with PET CBF imaging in subjects receiving vibrotactile stimulation (Fox et al. 1987). The postcentral responses were mapped onto a stereotactic atlas and the distribution was concordant with historic results obtained by direct cortical stimulation by Penfield and others.

We hypothesized that localization of neuronal activity associated with voluntary motor tasks might yield less spatial variability than previously reported motor studies in which cortical stimulation was evoked by electrophysiologi- 
cal techniques. A paradigm requiring accurate movements of the dominant first finger, arm, great toe, and tongue during CBF imaging was developed. In five subjects, matched and registered magnetic resonance imaging (MRI) studies were also available so that the distribution of neuronal activation along the precentral gyrus could be defined. With these registered studies, presented in three-dimensional perspective, the localization of motor areas relative to surrounding gyral anatomy could be examined. In addition, reproducibility studies were obtained to determine the variability of the magnitude and site of the maximal $\mathrm{CBF}$ response associated with voluntary movements of the first finger. Concomitant relative CBF responses in other cortical motor areas and the cerebellum will be addressed in a separate report.

\section{METHODS}

\section{Imaging}

Serial images of relative CBF were obtained in 12 normal subjects while they performed a set of motor tracking tasks. Each subject received six sequential intravenous bolus injections of 50 $\mathrm{mCi}$ of $\mathrm{H}_{2}{ }^{15} \mathrm{O}$ and underwent dynamic positron emission tomographic imaging with the Siemens/CTI 831-08 tomograph (Siemens, Hoffman Estates, IL). The device collects 15 contiguous planes, $6.75 \mathrm{~mm}$ thick, with an in-plane resolution of $13 \mathrm{~mm}$ full-width half-maximum in the final reconstructed images with $\mathrm{H}_{2}{ }^{15} \mathrm{O}$. Each emission image was corrected for attenuation by the use of a transmission image of the subject collected in the same position with a Germanium-68 ring source. Head immobilization was achieved with a custom foam mold (Smithers, Akron, $\mathrm{OH}$ ).

Estimates of relative CBF were based on a modified autoradiographic method (Herscovitch et al. 1983; Raichle et al. 1983). Five-second frames were collected for $2 \mathrm{~min}$, and the first frame showing the arrival of cerebral radioactivity was identified. From this time point the counts for the ensuing $70 \mathrm{~s}$ of time were summed and used to generate relative CBF images. Previous studies have demonstrated an optimal signal-to-noise ratio in difference images at approximately this scan duration (Mintun 1989; Grafton, unpublished data). Arterial sampling was not performed, so that subjects would have at least one untethered arm to perform the motor tasks. The final images represented relative images of tissue activity rather than absolute estimates of regional CBF. Relative CBF images demonstrate a near-linear relationship with actual CBF and provide a reasonable estimate of local CBF (Fox et al. 1984; Mazziotta et al. 1985a). The effects of global normalization were assumed to induce relative underestimations for local increases in CBF. Because global changes of CBF are usually $<10 \%$, the overall effect was assumed to be small and to cause a conservative error. That is, underestimates of relative blood flow responses.

\section{Subjects}

Twelve subjects (mean age $24 \mathrm{yr}, 11$ male, 1 female) were studied. All were drug free, without any history of nervous system disease or injury, and all were normal on detailed neurologic examination. Five of the subjects also had MRI imaging. These MRI scans were interpreted as normal by an experienced neuroradiologist. Handedness was determined with a standardized questionnaire and the dominant side was used for performance of tasks ( 10 right-handed, 2 left-handed subjects). All subjects were studied after giving informed consent in accordance with the UCLA Human Subject Protection Committee.
Tasks

A series of motor tasks were performed in response to a single stimulus. The subjects were presented with a randomly moving 0.5 -cm target displayed on a 13-in color video monitor (Macintosh IIcx). The monitor was placed directly in front of the subject so that it could be touched comfortably with an outstretched arm. The visual fields peripheral to the computer screen were shielded to eliminate potential visual distractions. The target, moving in a fixed window of $6 \times 8 \mathrm{~cm}$, had a velocity of $6 \mathrm{~cm} / \mathrm{s}$ and changed direction randomly (an average of 200 times/min). The scanning was performed in a dimly lit room where the only ambient sound was from a tomograph fan. In the control studies, the subjects were asked to follow the movement of the target with their eyes only.

In the first motor paradigm, the subjects were instructed to track the moving target with the index finger of their dominant hand. The rotation of axis was about the first metacarpophalangeal joint and average displacement of the fingertip per movement was $<2$ $\mathrm{cm}$. The hand was lightly braced against the video terminal for stability, and the subjects were allowed to practice the tracking until only the first finger of their dominant hand slid smoothly over the target without any jumping movements of the finger or concomitant movements of the arm. This was the only task in which the video monitor was touched.

In the second motor paradigm, the subjects held their dominant hand in a fist and pointed at the target with the first metacarpal head and with their arm held in extension. Thus the axis of rotation was at the shoulder joint.

In the third motor paradigm, the subjects followed the moving target with the great toe ipsilateral to their dominant hand. The leg was completely relaxed at the hip and knee and lay in extension. Flexion and extension of the great toe and rotatory movements at the ankle allowed for accurate tracking. Because the computer screen was closer to the subject's eyes than the toe, the subjects did not have visual feedback from projection cues to confirm accurate spatial displacement of the toe in reference to the moving target on the computer screen.

In the last paradigm, the subjects tracked the target with their extended tongue. They were coached not to use their lips, face, or limbs in concert with the tongue movements. As with the toetracking paradigm, visual projection cues and somatosensory feedback were not present.

Subjects practiced the motor tasks for $\sim 5 \mathrm{~min}$ before the PET imaging. Each task was then performed for $2 \mathrm{~min}$, beginning with the bolus injection of $50 \mathrm{mCi}$ of $\mathrm{H}_{2}{ }^{15} \mathrm{O}$ and dynamic imaging. The subjects were monitored by visual inspection for any axial or appendicular movements other than the designated limb or digit. They were also rated for their accuracy in following the tracking target and rewarded monetarily for their qualitative performance.

Two block design protocols were performed. In the first, termed "reproducibility studies," five subjects performed the finger-tracking experiment in three sessions alternating with three sessions of the control paradigm to determine the reproducibility of the sensory-motor cortical response. In the second block of experiments, termed "homunculus studies", seven subjects performed, in the following order, the index finger, control, arm, tongue, control, and toe-tracking tasks to define the spatial distribution of the sensory-motor homunculus. The homunculus task sequence was selected so that the task most likely to cause inadvertent head movement (toe tracking) would be performed last. Control tasks were interspersed between the motor tracking tasks so that if a subject moved at any time throughout the session there would still be pairs of stimulus and control for comparison. Because the subjects had adequate time to practice all of the tasks in random order before imaging, we assumed that the effect of not counterbalancing the stimuli would be negligible. 


\section{Image analysis}

Contours from each $\mathrm{H}_{2}{ }^{15} \mathrm{O}$ count image were obtained with a threshold algorithm to define global brain regions of interest. All studics from each individual were normalized to a common global CBF value. Percent change (PC) in the images, where $P C=[($ stimulus - control)/control] $* 100$, were calculated within each subject on a pixel-by-pixel basis. Only positive changes of CBF were analyzed for this report. Each PC image was inspected for artifacts indicative of misregistration because of head movement. Artifacts appeared as extraaxial rims of activity along the cortical surface or as a halo over the dorsalmost brain. Any such studies were excluded from further analysis.

For the reproducibility studies, the first control session from each subject was subtracted from the other five studies. PC in CBF images were evaluated with a SUN 4/60 computer with the Analyze ${ }^{\Theta}$ program (Mayo Clinic, MN). The spatial location and magnitude of the site of maximal response in the sensory-motor cortex was measured for each of the three tracking studies per individual.

For the homunculus experiments, a control study was subtracted from each of the tracking tasks. The maximum site of activation in the primary motor cortex was localized and the peak percent increase of relative CBF determined.

Functional localization was examined with the use of three anatomic references. In the first reference system, five of the six subjects underwent MRI scanning with an inversion recovery protocol [echo time $(\mathrm{TE})=30 \mathrm{~ms}$, inversion time $(\mathrm{TI})=300 \mathrm{~ms}$, and recovery time $(\mathrm{TR})=1275 \mathrm{~ms}$ ] that generated 30 contiguous planes of data with an interslice distance of $4.4 \mathrm{~mm}$ and in-plane resolution of $1 \mathrm{~mm}$ (Fonar Systems, 0.35 Tesla, Melville, NY). Contours at the skin surface obtained from the MRI and the PET transmission scan sets were registered and aligned (Pelizzari et al. 1989). The PET PC images were then resliced to match the MRI image set to allow for accurate anatomic localization of intrasubject sensory-motor cortical responses. An unsubtracted $\mathrm{H}_{2}{ }^{15} \mathrm{O}$ study from each subject was also resliced and compared with the MRI scan to verify the accuracy of the registration and alignment between the two study modalities. To do this, we compared the location of anatomic references that are readily visualized in a $\mathrm{H}_{2}{ }^{15} \mathrm{O}$ study, such as the midline, the calcarine cortex, the grey matter boundary, the thalamus, and the striatum, to the MRI study. Studies without concordance were excluded $(n=1)$. After registration, alignment, and reslicing of the PET studies, the images were processed with the Analyze program. Three-dimensional volume-rendered images of the grey/white border of the MRI study were created. Color PET studies were then added at varying thresholds above a given $\mathrm{PC}$ in relative blood flow. The grey/white border was used for the MRI studies to allow for the visualization of deep sites of focal activation within sulci.

In the second anatomic reference, each PC image was sliced to generate a $2.5-\mathrm{cm}$ thick coronal section that passed through the dorsal and ventral extent of the central fissure. To do this, we fit the anterior-posterior commissural (AC-PC) line on the PET studies or, when available, directly on the MRI scans by the use of the "Explorer" program and the Apple Macintosh computer (software developed by O. Ratib, G. Porenta, and W. Kuhle, U.C.L.A. Division of Nuclear Medicine and Biophysics, Los Angeles, CA) (Friston et al. 1989; Ratib and Huang 1989). A section forming an angle of $\sim 112^{\circ}$ relative to the AC-PC line (Matsui and Hirano 1978 ) and having a thickness of $2.5 \mathrm{~cm}$ consistently included foci of activation for toe, finger, and tongue responses in all cases. The point maxima for each stimulation were then mapped onto the coronal section. A radial with origin at the roof of the third ventricle and passing through each site of activation in the motor cortex was drawn on the coronal section containing the motor cortex. The solid angle that each of these maxima formed relative to the midline and a radial centered on the roof of the third ventricle at the fornix were then measured. The roof of the third ventricle could be located directly on MRI studies estimated on unsubtracted PET $\mathrm{H}_{2}{ }^{15} \mathrm{O}$ images. The accuracy of finding this point on the unsubtracted $\mathrm{H}_{2}{ }^{15} \mathrm{O}$ studies was determined. Unsubtracted $\mathrm{H}_{2}{ }^{15} \mathrm{O}$ studies were registered, aligned, and resliced to match each subject's MRI scan. The roof of the ventricle was then identified on the unsubtracted coronal $\mathrm{H}_{2}{ }^{15} \mathrm{O}$ studies without the aid of the MRI scan. The same site was identified on the MRI. The site agreed to within $2 \mathrm{~mm}$ for the five subjects with registered and aligned MRI scans.

In the third anatomic reference, the coronal sections were again generated. A circumferential curve was drawn across the surface of the gray matter, along a meridian that paralleled the central fissure. The distance along this meridian, from the lateral and interhemispheric fissures to the maximum site of activation, was measured for each study. This circumferential distance is analogous to the distance that could be practically measured intraoperatively with a tape measure. The fraction of the distance from the lateral fissure to each site, relative to the distance to the interhemispheric fissure, was calculated.

Region-of-interest templates were drawn on unsubtracted relative CBF images or, when available, the matched and registered MRI scans over the right and left caudate, putamen, and thalamus. PCs in relative CBF in these regions were then calculated. For brain volumes spanning multiple image planes, the average relative increase in $\mathrm{CBF}$, weighted for the area in each plane, was calculated.

\section{RESULTS}

\section{Reproducibility studies}

Five subjects performed the index finger tracking task in three serial sessions. These studies were then compared with control studies during which the subjects tracked the same moving target with their eyes alone. In all cases, clear foci of activation could be delineated in the superior primary sensory-motor cortex, the premotor cortex, the supplementary motor area contralateral to the moving finger, and the anterior superior cerebellar hemisphere ipsilateral to the hand performing the task. The pixel of maximal response in the sensory-motor cortex was identified for each study. The average maximal increase in relative $\mathrm{CBF}$ was $41.1 \pm 8.9 \%$ (SD) with a coefficient of variation of $22 \%$ across all individuals and $9 \%$ within individuals. There was no systematic trend in the response between the first and third study across all individuals to suggest a practice effect or habituation.

Previous PET CBF studies have demonstrated that the site of a maximal CBF response may be determined with a high degree of spatial accuracy (Fox et al. 1986). For our tomograph, the ability to detect a shift in the location of a maximal response between sequential PET difference images is determined principally by the pixel size of 1.7 $\mathrm{mm}$. An additional methodological question is the degree to which two maxima may be distinguished within a single PET difference image. The power to discriminate two adjacent foci will depend in part on the variability of the spatial location of a CBF response. For a given individual, the variability is secondary to physiological shifts of the CBF peak (dependent on the task design), and methodological artifacts such as subtle head movement. To estimate these two variance components, the maximal responses in the motor cortex from each individual who performed the reproducibility task were localized. The geometric center between 
the CBF motor responses obtained in three scans (relative to a single control) were calculated, and the distance between the centroid and each of the three individual peak pixel values were determined. From these distances a $95 \%$ confidence limit within which a maximal site of activation would be expected to occur could be defined. Because the range for this distance varied from 0 to only positive values, a one-tailed limit was derived. Within subjects this limit was determined to be a sphere of $5.4 \mathrm{~mm}$ radius. Thus, two maxima separated by $>5.4 \mathrm{~mm}$ could be considered to be distinct sites of activation after accounting for physiological variability of the $\mathrm{CBF}$ responses and subtle head movement artifact.

For each subject a control study was subtracted from a second control study and the resultant PC image was processed at each of the cutoff threshold values and rendered in three-dimensional perspective (Fig. 1). For these control studies, no increased relative blood flow $>10 \%$ and $>10$ $\mathrm{mm}^{3}$ was observed.

\section{Homunculus studies}

All subjects demonstrated focal increases of relative CBF that followed the classic sequential somatotopic representation of the motor cortex as defined by Penfield (Penfield and Boldrey 1938). BFRs secondary to toe movements extended from the interhemispheric fissure to the dorsolateral surface, with the peak most commonly on the dorsolateral surface. Responses for arm and finger movements were approximately two-thirds of the distance from the lateral fissure to the interhemispheric fissure, and those of tongue movements were close to the lateral fissure (Fig. 2).

Arm and first finger tracking tasks caused superimposable areas of increased relative $\mathrm{CBF}$ along the motor cortex. The point maximum for the two tasks were indistinguishable (based on a $95 \%$ CI derived from the reproducibility studies). The mean circumferential distance of these maxima from the lateral fissure, measured on a meridian parallel to the central fissure was $6.0 \pm 0.6(\mathrm{SD}) \mathrm{cm}$. The propor- tional distance, relative to the distance from the lateral fissure to the interhemispheric fissure, was $54 \pm 7 \%$ (SD).

In three of the four subjects who performed tracking with the toe and ankle, the foci of increased relative $\mathrm{CBF}$ were consistently localized to the dorsolateral surface of the primary motor cortex rather than the interhemispheric fissure. In the fourth, the focus was at the border of the dorsolateral and interhemispheric cortex. The average circumferential distance from the interhemispheric fissure to the maximum relative increase of $\mathrm{CBF}$ on the dorsolateral surface was $1.0 \pm 0.6(\mathrm{SD}) \mathrm{cm}$. The proportion of this distance relative to the length of the central sulcus was $91 \pm 7 \%$ (SD).

Tongue movements were always associated with bilateral relative blood flow increases of $>15 \%$. The average circumferential distance from the lateral fissure to the tongue area was $2.1 \pm 0.4(\mathrm{SD}) \mathrm{cm}$. The proportion of this distance relative to the length of the central sulcus was $18 \pm 2 \%(\mathrm{SD})$.

Because of a large variability in local gyral anatomy and potential inaccuracy in measuring the circumferential distance from the lateral and interhemispheric fissures, we sought to establish a localization schema for the maximal sites of activation in the motor cortex that was independent of surface landmarks. The solid angle that each focus of $\mathrm{CBF}$ change subtended relative to the midline was measured. From these measures a 95\% CI of two angles between which a focal site of activation would be expected to occur was defined (Fig. 3).

The maximum relative $\mathrm{CBF}$ response was used to estimate the magnitude of neuronal activity for each of the homuncular tasks, summarized in Table 1 . No significant differences between the homuncular tasks for this measure were present. Movements of the toe were associated with the greatest increase in relative CBF. Although the maximal sites of increased relative CBF for the arm and finger tracking task were indistinguishable, there was a trend toward a greater increase of relative $\mathrm{CBF}$ in the finger tracking task $(P=0.08$, Student's $t$ test). Movement in the finger task was predominantly across the metacarpophalangeal joint whereas in the arm tracking task the movements were cen-

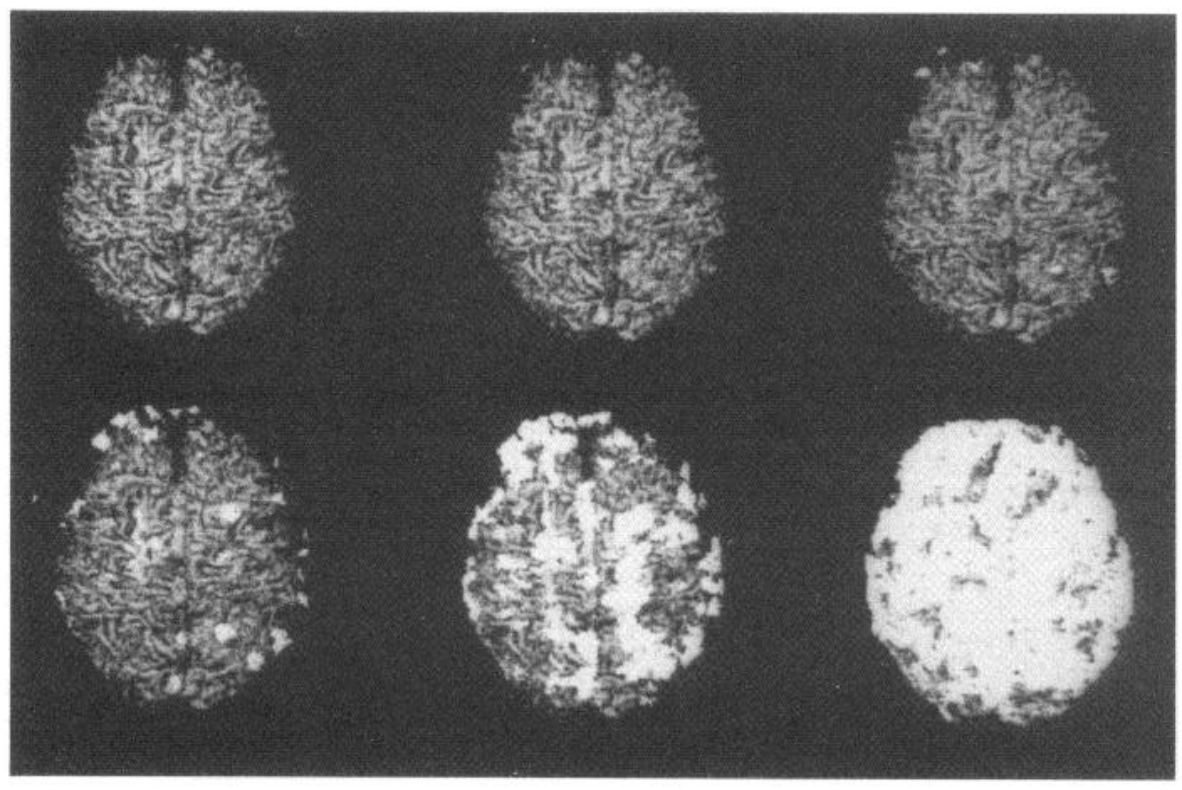

FIG. 1. Example of a percent change of relative blood flow between 2 control studies in which the subject tracked a moving target with eyes only. The PET images have been matched and registered to the same subject's MRI scan and displayed as a 3-dimensional representation, vertex view with frontal lobe at the top of the image. MRI gray matter has been removed to allow examination of potential sites of activation in deeply infolded sulci. Cutoff values from 25 to $0 \%$ are shown, beginning top left. At $>10 \%$ threshold there are no large, contiguous areas of increased cortical relative blood flow. 


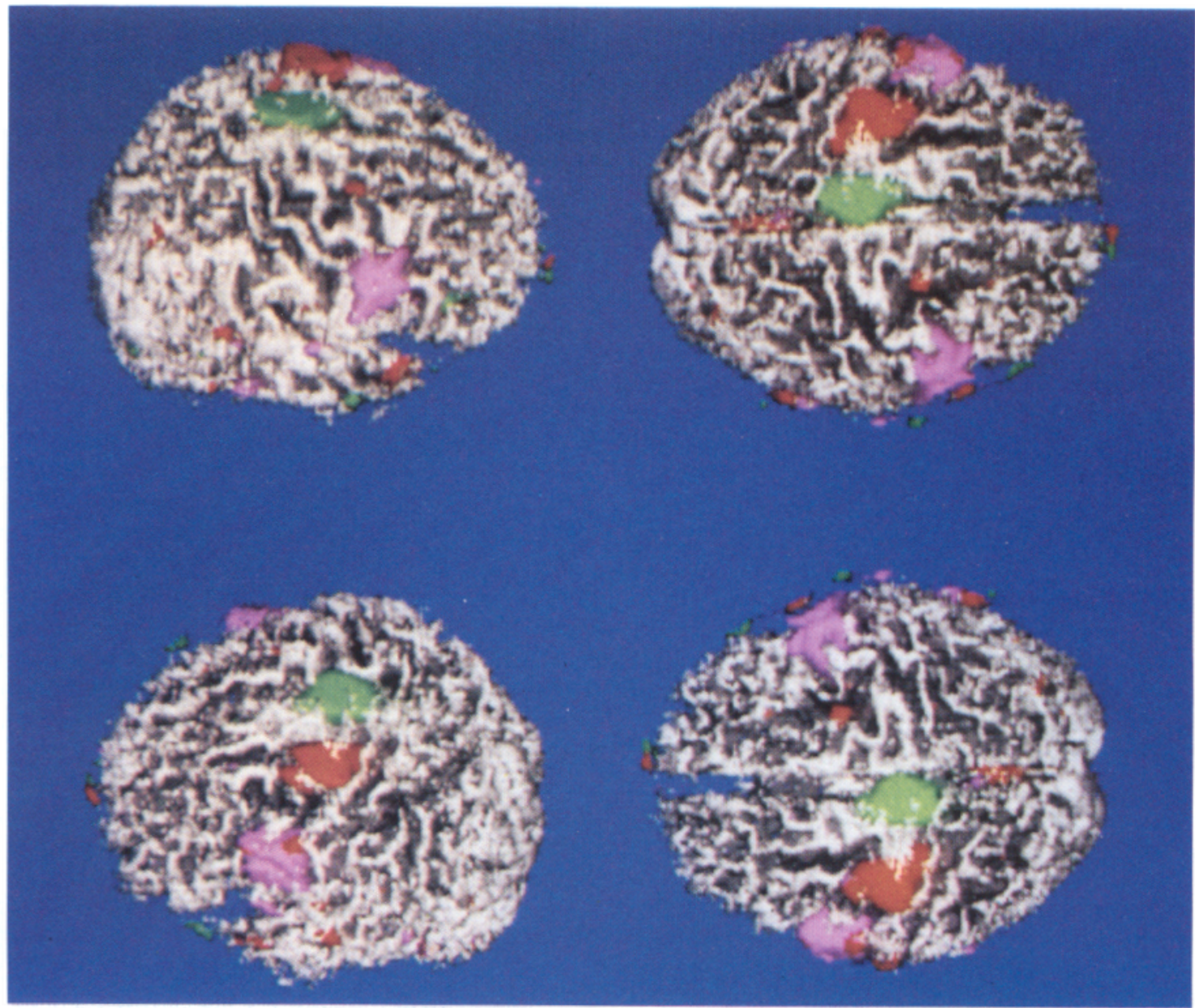

FIG. 2. The somatotopic representation of the motor cortex as demonstrated with relative cerebral blood flow imaging in a subject performing a series of motor tracking tasks. The relative blood flow increases above a 15\% threshold have been combined with the same subject's MRI study. Toe, finger, and tongue areas are identified in the precentral gyrus. Arm activation was in a similar area as finger movements (not shown). From clockwise top left, images are shown in the right superior oblique, superior (with frontal lobe facing right), superior (with frontal lobe facing left), and left superior oblique views.

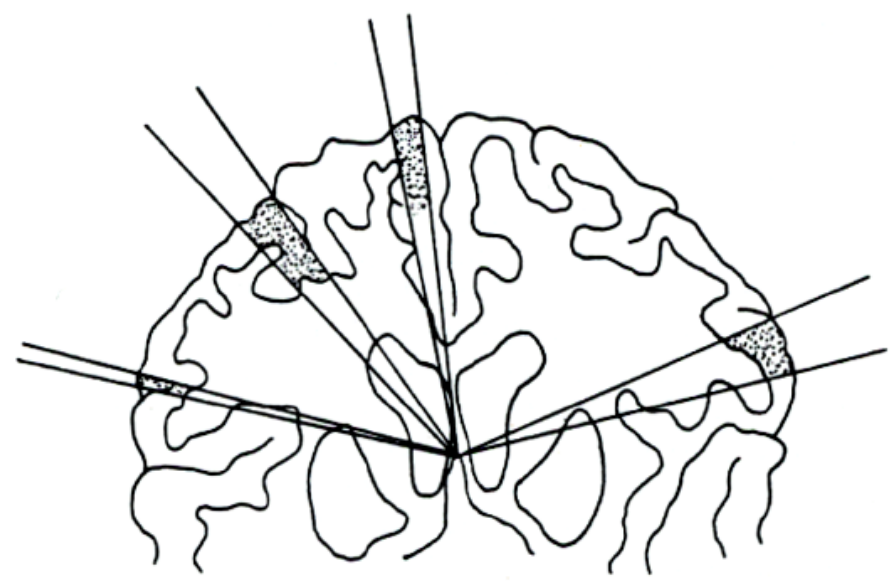

FIG. 3. Solid angles representing a $95 \%$ confidence interval within which the maximal site of activation of the primary motor cortex would be expected to occur for movements of the toe (mean, 83.5, 95\% CI:80.786.3 ), tongue (mean, $14.8,95 \% \mathrm{CI}: 14.0-15.6$ ), contralateral tongue (mean, 16.8, 95\% CI:12.0-21.7), and finger (mean, 53.0, 95\% CI:49.4-56.6). Angles from the population have been projected onto a coronal section from 1 subject that passes through the primary motor cortex. tered at the shoulder. Both tasks required sustentation to keep the arm in extension. Increases in relative blood flow secondary to tongue movement revealed no significant asymmetry in the peak change in flow.

Quantitative region-of-interest analysis of the right and left putamen, caudate, globus pallidus, and thalamus revealed no significant increase in relative blood flow associated with any of the tracking tasks. In addition, no later-

TABLE 1. Peak changes of relative CBF of the primary motor cortex in 12 individuals performing motor tracking tasks

\begin{tabular}{lrc}
\hline \hline Motor Tracking Task & $n$ & $\begin{array}{c}\text { Maximum Relative } \\
\text { CBF Increase }\end{array}$ \\
\hline Arm & 5 & $30.4 \pm 11.1$ \\
Finger & 12 & $37.3 \pm 12.5$ \\
Tongue & 6 & $34.0 \pm 9.1$ \\
Contralateral tongue & 6 & $35.3 \pm 6.5$ \\
Toe & 4 & $40.3 \pm 13.3$ \\
\hline
\end{tabular}

Values are means $\pm \mathrm{SD}$; changes are relative to a control study. 
alizing changes in CBF were noted in these subcortical structures.

Five subjects had matching MRI studies, and, in all cases, sites of focal activation could be related to regional gyral anatomy on an individual basis. Correlations between subjects were difficult because of significant variability in gyral anatomy. In normal subjects, the central sulcus makes two curves as it courses from the interhemispheric to lateral fissure. The arm and finger areas were consistently localized to an area anterior to the dorsal curve (Fig. 3). This localization was in agreement with the findings from direct cortical stimulation performed by Penfield (See Figs. 12 and 14, Penfield and Boldrey 1938). Activation secondary to tongue movements was always close to the lateral fissure and not adjacent to any specific gyri. Toe activation was always close to, but typically not within, the medial hemispheric cortical surface. This site was also in agreement with Penfield's original finding (see Penfield and Boldrey 1938, Figs. 5 and 16). Although toe responses could occur in the medial hemispheric surface, the majority of his cases and the maxima from three of four PET studies reported herein were localized to the dorsolateral surface of the precentral gyrus. In no subject could a second discrete focus of increased relative $\mathrm{CBF}$ be detected in the postcentral gyrus to suggest significant somatosensory response to any of the tracking paradigms.

There was a large degree of variability in the distance from the maximal site of increased relative CBF in the precentral gyrus to the central fissure, measured in the anterior-posterior direction. Similar variability was present for the depth of the maximum site. In reviewing all of the tracking tasks, the point maxima were distributed equally between sites located superficially at the surface of gyri and deep within sulci.

\section{DISCUSSION}

In this study we have demonstrated a predictable distribution for the sites of neuronal activation in the primary motor cortex induced by voluntary movements of the toe, finger, arm, and tongue. The pronounced magnitude of relative CBF responses allowed for accurate localization in every subject without intrasubject averaging. Reproducibility studies validated the use of the maximum site of increased relative blood flow as a reliable localizing variable for determining focal sites of neuronal activation. With this parameter, the somatotopic representation of the motor cortex could be examined with respect to several anatomic references.

Both the circumferential and angular displacement measurements revealed remarkably low topographical variation between subjects for the localization of the different motor areas along the precentral gyrus. For tongue, finger, arm, and toe tracking, responses combined with the MRI localization were in close agreement with the electrophysiological stimulation studies reported by Penfield. The span of cortex along which a site of increased relative CBF would be expected to occur during performance of a finger tracking task was shorter in our study $(<1 \mathrm{~cm})$ than the span of cortex in which stimulation would be expected to induce finger or hand movements $(5.5 \mathrm{~cm})$. This discrepancy was less apparent for the toe and tongue tracking task. The differences between the two localization methods may, in part, be secondary to the effect of surface stimulation compared with volitional movement. There is an unpredictable spread of surface electrical stimulation through cortical tissue with electrophysiological techniques. None of our subjects demonstrated pathological abnormalities that might distort normal topology or, potentially, induce functional reorganization along the precentral gyrus.

The location of motor responses associated with finger movements were qualitatively similar in the vertical and lateral dimensions to previously reported somatosensory responses in the postcentral gyrus (Fox et al. 1987). In that study, mapping of the tongue area was not performed, so a comparison with our tongue motor area responses cannot be made. In contrast, the location of toe motor responses on the matched and registered MRI scans (centered in the deep, dorsolateral cortex) was notably different from toe somatosensory responses. With vibrotactile stimulation, responses were identified in the depth of the interhemispheric fissure, close to the cingulate gyrus. In comparing the two studies, we cannot answer definitively whether homuncular motor and sensory responses are mirror foci located across the central fissure, because of the use of different localization techniques. The two studies suggest that a paradigm using paired motor and sensory stimuli would successfully provide sufficient anatomic detail to resolve this interesting question.

Overall, the most consistent anatomic reference was based on a radial coordinate system centered on the roof of the third ventricle and mapped onto a coronal section that was parallel to and contained the central fissure and precentral gyrus. In some ways this is not surprising, because this approach reduces a three-dimensional problem to two dimensions and does not measure the depth (i.e., within or outside of sulci) of a site of increased relative CBF in the primary motor cortex. This may have an advantage, given the variability of the depth of increased relative CBF measured with PET.

The basis for the relative invariance of the angular displacement of the activation sites when viewed in coronal section is not known. One possible explanation is that it is related to the normal pattern of corticogenesis during early development of the nervous system. Embryological studies have demonstrated that there is a radial organization of growth cones emanating from the ependymal matrix to the cerebral mantel (Rakic 1988). It may be that functional localization, at least for the primary motor cortex, is more dependent on the angular displacements of these growth cones during development than the later gyral formation and sulcal infolding. This is supported in part by our finding of a wide variety in the pattern of folding of the precentral gyrus, the inconsistent depth of foci into the central sulcus, and the unpredictable distance of foci from the sulcus onto the surface of the gyrus.

Interpretation of the results for the finger and arm tracking tasks were confounded by the matching foci of maximal activation for these two tasks. There are several tenable explanations for this result. There could be congruent foci of 
increased blood flow if the PET method of relative CBF imaging was inadequate for distinguishing two sites of neuronal activation with sufficient resolution. Prior studies have shown that sites $<2 \mathrm{~mm}$ apart may be distinguished when the centroid of the site of activation from serial images is used for localization (Fox et al. 1988). With current-generation PET tomographs providing contiguous axial sampling and plane thicknesses of $\leq 6.75 \mathrm{~mm}$, it is unlikely that the identical results are secondary to insufficient spatial resolution of the imaging device.

In addition to tomograph resolution, physiological variability, and, potentially, movement artifact must also be considered. From our reproducibility studies we determined that two foci of responses must be separated by distance of $\geq 5.4 \mathrm{~mm}$ to be considered separate. It may be that arm and finger motor neuron groups exist closer than 5.4 $\mathrm{mm}$. However, this is unlikely on the basis of previously reported nonhuman primate and human electrophysiological studies (Asanuma 1989). Both tasks required the subject to maintain an extended posture of the arm. The maximal site of activation, representing the sum of overlapping foci along the motor cortex, may be dominated by the motor neuron pool maintaining arm sustentation. Other hypotheses can be explored. It may be that the common site of increased relative blood flow is related to activation of higher-order neurons regulating the displacement of the distal limb rather than the activation of corticospinal motor neurons of the final common pathway that are also required for the task execution. That is, the relative changes in $\mathrm{CBF}$ are a reflection of the movement or direction required by the tasks rather than the motor neurons executing the tasks (Georgeopolis et al. 1986; Green and Odom 1986).

The finger tracking task consistently induced greater increases of blood flow than the arm task. This could have been secondary either to differences in the number of motor neurons activated or to increased afferent somatosen- sory input with the finger task because the screen was being touched with the finger. Because these were relative studies calculated after global normalization, artifactual depression of blood flow changes secondary to global CBF increases cannot be ruled out. The paradigm presented in this report forms a basis that may be refined for the comparison of finger versus proximal arm movements.

Within subjects, we found the peak response to vary by $9 \%$. The variability may, in part, be related to alterations in the pattern of recruitment of auxiliary muscle groups involved in performing a motor task across trials. Variability of the response also may be secondary to effects of plasticity or the use of different representations of the hand area within the cortex. Differences between trials could be secondary to a conscious effort on the subject's part (change in strategy) or an unconscious effect related to practice, improved efficiency, boredom, or decreased effort. Variable effects of practice, progressive boredom, distraction, or improved efficiency are possible, although there was no systematic increase or decrease in relative CBF across three consecutive trials. We did not measure motor performance, and thus a direct comparison between changes of blood flow and motor activity could not be examined.

Changes of relative CBF during motor performance may occur potentially at the microscopic, macroscopic, and hemispheric level. By decreasing the threshold of the PC images, we could demonstrate that the maximal site of activation is not present in isolation. Rather, it is embedded in local cortical tissue with concomitant blood flow increases (Fig. 4). As the threshold for the observable change in CBF was reduced, there was progressive delineation of other components of the motor system, including the premotor cortex and supplementary motor areas as well as the posterior parasagittal parietal association cortex. With the threshold approach, discrimination of the functional boundary between the anatomically contiguous premotor and primary motor area was somewhat arbitrary for the finger and

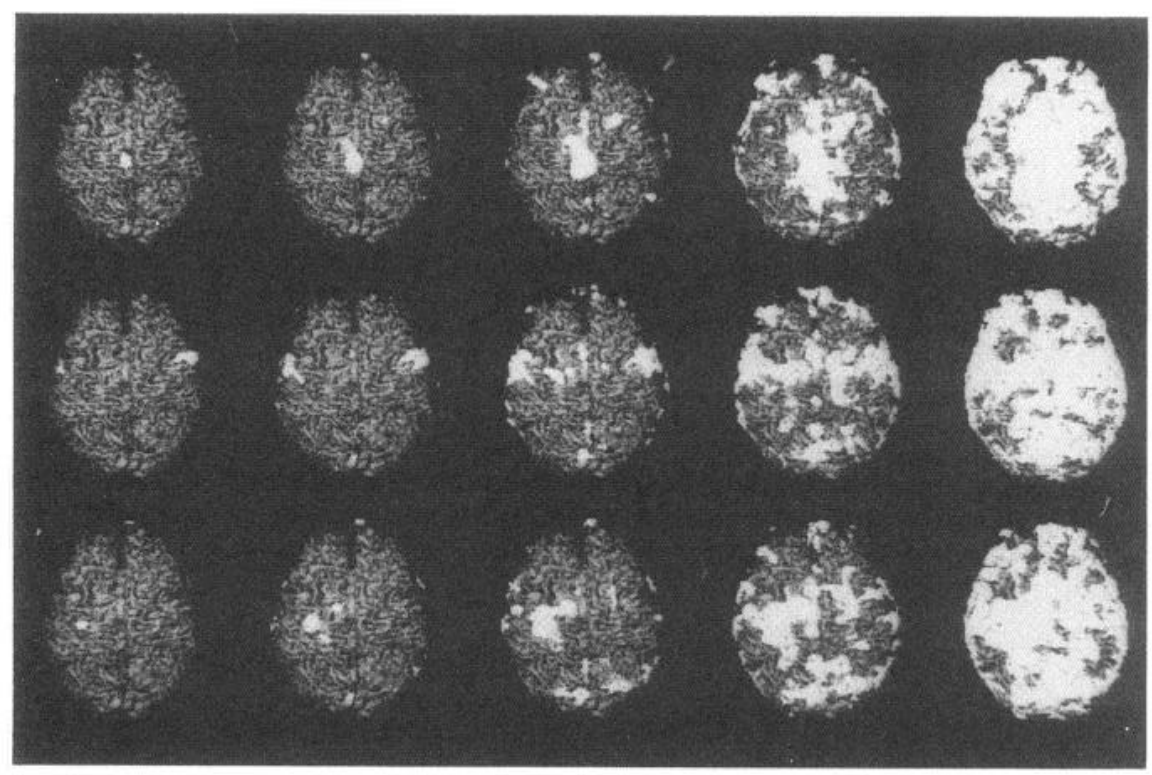

FIG. 4. The distributed responses of the motor system determined with relative CBF imaging. As the threshold for a percent change of relative blood flow is reduced from 25 to $0 \%$ (column 1 to 5 ), premotor cortex, supplementary motor area, and bilateral primary motor cortex are visualized. The 3 rows are from a subject performing visually guided movements with the toe (top), tongue (middle), and index finger (bottom). Vertex view with frontal lobe at the top of the image. 
arm tracking tasks. This presents a considerable challenge for quantitation of discrete or circumscribed brain regions and merits further development.

In contrast to the cortical responses, basal ganglia nuclei failed to demonstrate any significant increases in regional relative CBF. Previous authors have demonstrated striatal and thalamic responses in subjects performing a task requiring digit tapping in a complex pattern (Roland et al. 1982). The absence of these basal ganglia responses in our study may be a function of the paradigm. Our task required unpatterned tracking movements without significant somatosensory feedback.

An implicit assumption of this study was that regional changes of relative CBF were linearly proportional to increased neuronal activity. Although this assumption has never been tested directly, a large series of PET CBF studies have demonstrated a close association between sensory stimuli or behavioral tasks and functional activation as assessed by blood flow and glucose and oxygen metabolism (Fox and Raichle, 1985, 1986; Ginsberg et al. 1988). Changes in the intensity of both somatosensory or visual stimuli have caused proportional and linear alterations in local CBF (Fox and Raichle 1985; Fox et al. 1987). These prior studies have, in part, validated the PET blood flow imaging technique as a tool for the functional localization of neurobehavioral tasks. Comparative studies that combine electrophysiological and PET imaging methods in nonhuman primates or humans undergoing cortical mapping are needed to characterize the spatial and temporal features that couple flow and neuronal activity.

The current results have demonstrated the utility of assessing the functional organization of the motor system throughout the entire brain, viewed as a whole, with PET. If combined PET and MRI studies are available, then localization of the motor cortex with respect to surface anatomy of the brain or skull may be made directly. If MRI is not available, then the angular displacements derived from the radial-based localization approach can also be transformed so that surface landmarks, such as skull anatomy, may be used as a reference. This may have particular utility in the operating suite.

We acknowledge D. K. Mahoney, O. Ratib, G. Porenta, and W. Kuhle for software development; L. Carr for preparation of the manuscript; and L. Griswold for preparation of illustrations.

This work was supported in part by Department of Energy cooperative agreement \#DE-FC03-87ER60615 and National Institute of Mental Health Grant RO1-MH-37916.

Address for reprint requests: S. T. Grafton, Division of Nuclear Medicine and Biophysics, B2-085H Clinical Health Sciences, University of California at Los Angeles School of Medicine, 10833 LeConte Blvd., Los Angeles, CA 90024.

Received 12 October 1990; accepted in final form 10 May 1991.

\section{REFERENCES}

Asanuma, H. The Motor Cortex. New York: Raven, 1989.

FER RIER, D. Experiments on the brain of monkeys. Proc. R. Soc. Lond. B Biol. Sci., 23: 409-430, 1875 .
Ferrier, D. The Functions of the Brain. New York: Putnam, 1876, p. $139-145$.

Fox, P. T., Burton, H., AND Raichle, M. E. Mapping human somatosensory cortex with positron emission tomography. J. Neurosurg. 67: 34-43, 1987.

Fox, P. T., Mintun, M. A., Raichle, M. E., ANd Herscovitch, P. A noninvasive approach to quantitative functional brain mapping with $\mathrm{H}_{2}{ }^{15} \mathrm{O}$ and positron emission tomography. J. Cereb. Blood Flow Metab. 4: 329-333, 1984.

Fox, P. T., Mintun, M., Raichle, M. E., Miezin, F. M., Allman, J. M., AND VAN EsSEN, D. C. Mapping human visual cortex with positron emission tomography. Nature Lond. 323: 806-809, 1986.

Fox, P. T., Mintun, M. A., Reiman, E. M., and Raichle, M. E. Enhanced detection of focal brain responses using intersubject averaging and change-distribution analysis of subtracted PET images. J. Cereb. Blood Flow Metab. 8: 642-653, 1988.

FoX, P. T. AND RAICHLE, M. E. Stimulus rate determines regional brain blood flow in striate cortex. Ann. Neurol. 17: 303-305, 1985.

FoX, P. T. AND RAICHLE, M. E. Focal physiological uncoupling of cerebral blood flow and oxidative metabolism during somatosensory stimulation in human subjects. Proc. Natl. Acad. Sci. USA. 83: 1140-1144, 1986.

Friston, K. J., Passingham, R. E., Nutt, J. G., Heather, J. D., Sawle, G. V., AND FRACKOWIAK, R. S. J. Localisation in PET images: direct fitting of the intercommissural (AC-PC) line. J. Cereb. Bld. Flow Met. 9: 690-695, 1989.

FrITSCH, G., HitzIG, E. Ueber die elektrische Erregbarkeit des Grosshirns. Arch. Anat. Physiol. Wiss. Med. 37: 300-332, 1870.

Georgopoulos, A. P., Schwartz, A. B., AND Kettner, R. E. Neuronal population coding of movement direction. Science Wash. DC 233: 1416-1419, 1986.

Ginsberg, M. D., Chang, J. Y., Kelly, R. E., Yoshit, F., Barker, W. W., INGENITO, G., AND BoOTHE, T. E. Increases in both cerebral glucose utilization and blood flow during execution of a somatosensory task. Ann. Neurol. 23: 152-160, 1988.

GREEN, M. AND ODOM, J. V. Correspondence matching in apparent motion: evidence for three-dimensional spatial representation. Science Wash. DC 233: 1427-1429, 1986.

Herscovitch, P., Markham, J., and Raichle, M. E. Brain blood flow measured with intravenous $\mathrm{H}_{2}{ }^{i} \mathrm{O}$. I. Theory and error analysis. J. Nucl. Med. 24: 782-789, 1983.

INGVAR, D. H. AND PHILIPSON, L. Distribution of cerebral blood flow in the dominant hemisphere during motor ideation and motor performance. Ann. Neurol. 2: 230-237, 1977.

Lauritzen, M., Henriksen, L., AND Lass, N. A. Regional CBF during rest and skilled hand movements by $\mathrm{Xe}-133$ inhalation and emission CT. J. Cereb. Blood. Flow Metab. 1: 385-389, 1981.

LEYTON, A. S. F. AND Sherrington, C. S. Observations on the excitable cortex of the chimpanzee, orangutan and gorilla. Q.J. Exp. Physiol. 11: $135-222,1917$.

Matsui, T. and Hirano, A. An Atlas of the Human Brain for Computerized Tomography. New York: Igaku-Shoin, 1978, p. 468-469.

MazziotTA, J. C. Human PET studies of local brain metabolism and blood flow during sensory and motor tasks: strategies and results. Proc. Am. Soc. Neuroimaging 8: 17, 1985.

Mazziotta, J. C., Huang, S. C., Phelps, M. E., Carson, R. E., MacDONALD, N. S., AND MAHONEY, D. K. A noninvasive positron computed tomography technique using Oxygen-15-labeled water for the evaluation of neurobehavioral task batteries. J. Cereb. Blood Flow Metab. 5: 70-78, 1985a.

Mazziotta, J. C. AND Phelps, M. E. Human physiological imaging studies of local brain metabolism and blood flow: strategies and results. In: Brain Imaging and Brain Function. Proceedings of the Association for Research in Nervous and Mental Disorders, edited by L. Sokoloff. New York: Raven, 1985, 121-137.

Mazziotta, J. C., Phelps, M. E., ANd Wapenski, J. Human cerebral motor system metabolic responses in health and disease. J. Cereb. Blood. Flow Metab. 5 Suppl. 1: S213-S214, 1985b.

Mintun, M. A., RaICHLE, M. E., AND QuARLes, R. P. Length of PET data acquisition inversely affects ability to detect focal areas of brain activation (Abstract). J. Cereb. Blood Flow Metab. 9 Suppl. 1: 349, 1989.

ORGOGOZO, J. M. AND LARSEN, B. Activation of the supplementary motor area during voluntary movements in man suggests it works as a supramotor area. Science Wash. DC 206: 847-850, 1979. 
Orgogozo, J. M., Larsen, B., Roland, P. E., Melamed, E., and LasSEN, N. A. Further studies on the supplementary motor area in man with the rCBF method. Acta Neurol. Scand. 60 Suppl. 72: 8-9, 1979.

Pelizzari, C. A., Chen, G. T. Y., SPelbring, D. R., Weichselbaum, R. R., AND CHEN, C.-T. Accurate three-dimensional registration of CT, PET, and/or MR images of the brain. J. Comput. Assisted Tomogr. 13: 20-26, 1989.

Penfield, W. The Excitable Cortex in Conscious Man. Liverpool: Liverpool Univ. Press, 1958.

PENFIELD, W. AND BOLDREY, E. Somatic motor and sensory representation in the cerebral cortex of man as studied by clectrical stimulation. Brain 15: 389-443, 1938.

Penfield, W. AND JASPER, H. Epilepsy and the Functional Anatomy of the Human Brain. Boston, MA: Little, Brown. 1954.

RAKIC, P. Specification of cerebral cortical areas. Science Wash. DC 241: 170-176, 1988.

Raichle, M. E., Martin, W. R. W., Herscovitch, P., Mintun, M. A., AND MARKHAM, J. Brain blood flow measured with intravenous $\mathrm{H}_{215} \mathrm{O}$. II. Implementation and validation. J. Nucl. Med. 24: 790-798, 1983.

RATIB, O. AND HUANG, H. K. Calipso: an interactive software package for multimodalit medical image analysis on a personal computer. J. Med. Imaging 3: 205-216, 1989.

RolAND, P. E. Organization of motor control by the normal human brain. Hum. Neurobiol. 2: 205-216, 1984.

Roland, P. E., Larsen, B., Lassen, N. A., AND Skinhoj, E. Supplementary motor area and other cortical areas in organization of voluntary movements in man. J. Neurophysiol. 43: 118-136, 1980a.

Roland, P. E., Meyer, E., Shibasaki, T., Yamamoto, Y. L., and ThOMPSON, C. J. Regional cerebral blood flow changes in cortex and basal ganglia during voluntary movements in normal human volunteers. J. Neurophysiol. 48: 467-480, 1982.

Roland, P. E., Meyer, E., Yammamoto, Y. L., AND Thompson, C. J. Dynamic PET as a tool in neuroscience: functional brain mapping in normal human volunteers. J. Cereb. Blood Flow Metab. 1 Suppl. 1: S463-S464, 1981.

Roland, P. E., Skinhoj, E., Lassen, N. A., ANd Larsen, B. Different cortical areas in man in organization of voluntary movements in extrapersonal space. J. Neurophysiol. 43: 137-150, $1980 \mathrm{~b}$.

ShERrington, C. The Integrative Action of the Nervous System. New Haven, CT: Yale Univ. Press, 1906. 